\title{
15. OCCURRENCES OF AIR-FALL VOLCANIC ASH DERIVED FROM THE LESSER ANTILLES ARC AT LEG 78A DRILL SITES1
}

\author{
James H. Natland, Deep Sea Drilling Project, Scripps Institution of Oceanography²
}

\begin{abstract}
Air-fall volcanic ash recovered at Deep Sea Drilling Project Sites 541, 542, and 543 on and east of the toe of the Barbados Ridge delineate middle and late Miocene, early Pliocene, and Pleistocene-Quaternary pulses of explosive volcanism in the Lesser Antilles arc. The ash beds at Site 541 allow precise correlation of intervals repeated by a probable reverse fault at this convergent margin.
\end{abstract}

\section{INTRODUCTION}

This chapter briefly summarizes the occurrence of airfall volcanic ash at three sites $(541,542$, and 543) drilled during Leg 78A near the deformation front of the Barbados Ridge complex east of the Lesser Antilles arc (Fig. 1). The ashes are important for establishing the timing of explosive volcanism in the arc, heretofore inferred from geological relationships on the islands (dated only in a general way by sparse biostratigraphic data and radiometric ages) and from surface piston cores east of the arc that document Quaternary explosive eruptions (Sigurdsson et al., 1980). In addition, Site 541 demonstrates the usefulness of ash beds in determining precise vertical offsets along high-angle reverse faults at convergent margins such as this where accretion of sediments is occurring.

\section{OCCURRENCE AND LITHOLOGY OF ASHES}

Nearly 250 volcanic ash layers up to $7 \mathrm{~cm}$ thick were recovered in cores from three sites drilled during DSDP Leg 78A. Most of these ash layers were recovered at Site 541, near the toe of the Barbados Ridge complex (Fig. 1), where a section was cored continuously from the seafloor through Miocene sediments into a complex zone of deformation coinciding with an active décollement at this convergent margin (Site 541 report, this volume). Fewer ash beds were recovered at Site 542, which is only a few kilometers seaward of Site 541. Recovery of sediments of all types was limited here by discontinuous coring above the décollement. At Site 543, the reference site east of the deformation front of the Barbados Ridge (Fig. 1), a condensed Neogene section containing many ash beds overlies Paleogene and Upper Cretaceous pelagic clays, radiolarian muds, and igneous basement. Scattered ash beds occur below the Miocene into the lower Eocene, but these are much more altered to clays than the younger ashes.

Separate ash occurrences, their age, color, and thickness, are listed in Table 1 . The thicker ashes commonly

\footnotetext{
${ }^{1}$ Biju-Duval, B., Moore, J. C., et al., Init. Repts. DSDP, 78A: Washington (U.S. Govt. Printing Office).

2 Address: Deep Sea Drilling Project, Scripps Institution of Oceanography, A-031, La Jolla, CA 92093 .
}

have flat bases and bioturbated tops (Fig. 2). Some ashes are gray, others nearly black. Many have high concentrations of plagioclase, lesser quartz, and minor pyroxenes and opaque minerals owing to density-sorting processes in the atmosphere (e.g., Sigurdsson et al., 1980). None shows evidence of being turbidites or even has significant grading of the type that can also be produced by atmospheric processes.

Coring deformation is severe in the topmost cores, especially in the upper $150 \mathrm{~m}$ cored at Site 543. Although the continuously cored section at Site 541 is less disturbed by coring, it is complicated by several faults, as documented by nannofossil biostratigraphy (Bergen, this volume). The faults are evidently high-angle reverse faults produced by the general processes of plate convergence, formation of a décollement further down in the section, and sediment accretion (Moore and Biju-Duval, this volume). The presence of ash beds is also obscured along shear zones that are particularly prominent in Cores $\mathbf{2 5}$ to 30 and 40 to 50 , the latter cores occurring in the vicinity of the décollement (Cowan et al., this volume). Where coring and tectonic disturbance are minimal, as elsewhere at Site 541, ash beds are useful as indicators of precise correlation of units offset along faults. Figure 3 shows the correlation of ash beds and nannofossil zones (Bergen, this volume) between portions of Tectonic Units $A$ and $B$, which are the principal repeated intervals above the décollement at Site 541 (see Site 541 report, this volume). The ash beds define a vertical offset of $158.5 \mathrm{~m}$ at this interval along the fault. This compares well with the estimate of $156 \mathrm{~m}$ calculated by Moore and Biju-Duval (this volume) by adding together individual offsets in the nannofossil biostratigraphy determined by Bergen (this volume).

All the ash beds at Sites 541 to 543 are air-fall ashes, because the bathymetric high of the Barbados Ridge complex isolates the sites from submarine pyroclastic turbidites originating at the arc. This high has existed since the Miocene (Westbrook, 1982). Sigurdsson et al. (1980) have shown that the Quaternary Roseau ash was dispersed in the upper atmosphere over $600 \mathrm{~km}$ east of its source on the island of Dominica (Fig. 1). I infer a similar general pattern of eruption, atmosphere dispersal, and submarine deposition for all the Leg 78A ashes, al- 


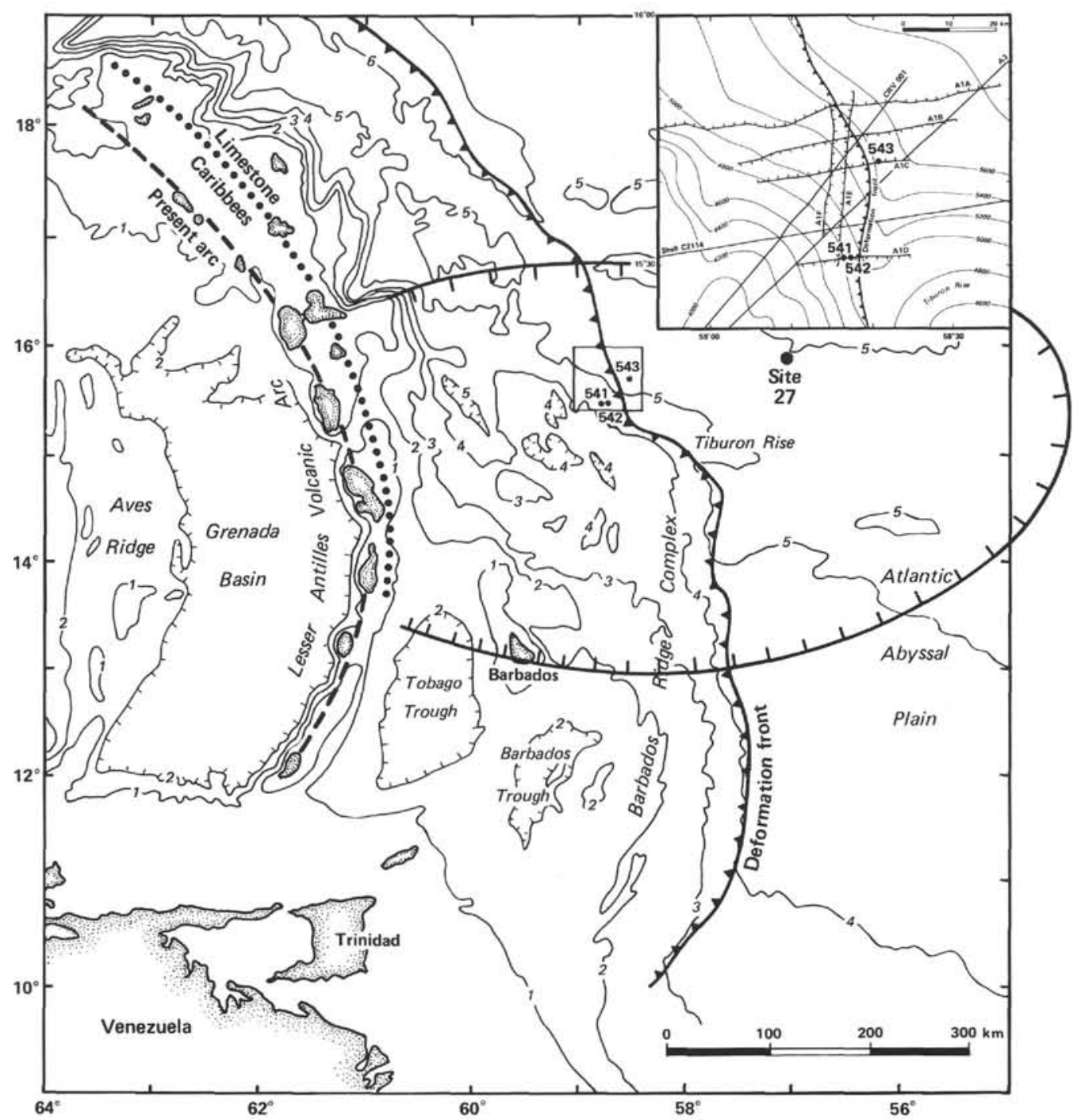

Figure 1. Location of the Leg 78A drill sites, the Lesser Antilles arc, and the Barbados Ridge complex. The divergence between the Limestone Caribbees and presently active arc at the northern end of the chain is also shown. The heavy dotted line gives the distribution of the 1-cm isopach of the Roseau ash east of its source at Dominica (after Carey and Sigurdsson, 1980).

though the direction and extent of ash dispersal are clearly dependent to some extent on climatic factors that have not been constant since the Miocene.

\section{TEPHROCHRONOLOGY}

A combination of information from Site 541 (postmiddle Miocene), Site 543 (pre-middle Miocene), and a Quaternary piston core $50 \mathrm{~km}$ east of Martinique (Sigurdsson et al., 1980) document the frequency of explosive eruptions from the Lesser Antilles arc (Fig. 4). Unfortunately, Quaternary cores from Leg $78 \mathrm{~A}$ are too disturbed by coring to provide information of this type.

Ages were assigned to ash beds using the nannofossil biostratigraphy of Bergen (this volume) for late Miocene and younger sediments, and the radiolarian biostratigraphy of Renz (this volume) for older sediments. Absolute ages for nannofossil zonal boundaries that are listed in Bukry (1975) and radiolarian zonal boundaries listed in Kling (1982) allowed interpolation of ash beds by depth. Fault repetition at Site 541 is accounted for by separately totalling the number of ash beds in each repeated nannofossil zone, and using the larger number (assuming that the discrepancy results from incomplete recovery and/or core disturbance in one of the intervals).

Major pulses of explosive Lesser Antilles volcanism occurred in the early and middle Miocene, the early Pliocene, and the Pleistocene-Quaternary, with possible minor pulses in the late middle Miocene and latest Miocene (Fig. 4). The early-to-middle Miocene pulse is documented from Site 543, which has been moving toward the Lesser Antilles as the North American Plate has converged on the arc. The ashes are thus probably underrepresented compared with locations such as Site 541, which have remained at a fixed distance closer to the arc since the Miocene (neglecting minor effects of section shortening related to accretion; Moore and Biju-Duval, this volume). Nevertheless, the three major pulses of explosive volcanism coincide with those documented by drilling adjacent to circum-Pacific arcs (cf., Kennett and 
Table 1. Occurrences of Volcanic Ash at Leg 78A drill sites.

\begin{tabular}{|c|c|c|c|c|c|}
\hline $\begin{array}{c}\text { Sample } \\
\text { occurrence } \\
\text { (core-section, } \\
\text { cm level) }\end{array}$ & \multicolumn{2}{|c|}{ Age-zone } & $\begin{array}{l}\text { Thickness } \\
\text { (cm) }\end{array}$ & Color & Comments \\
\hline \multicolumn{6}{|l|}{ Hole 451} \\
\hline $\begin{array}{l}2-5,120 \\
3-2,80 \text { and }\end{array}$ & & & $?$ & Black & Smeared out \\
\hline$a_{3-4,55}^{115}$ & & & $?$ & Gray & Blebs \\
\hline $\begin{array}{l}a_{3-4}, 55 \\
a_{4-3}, 140\end{array}$ & & & $2-3$ & Gray & Smeared out \\
\hline $\begin{array}{c}\mathrm{a}_{4-3}, 140 \\
4-7,50\end{array}$ & & Pseudo- & $2-3$ & Black & Arched \\
\hline $\begin{array}{l}4-7,50 \\
5-2,30\end{array}$ & & emilianna & $<\stackrel{?}{1}$ & Gray & $\begin{array}{l}\text { Bleb } \\
\text { Bioturbated }\end{array}$ \\
\hline $\begin{array}{r}5-2,30 \\
5-2,60\end{array}$ & $\begin{array}{c}\text { early } \\
\text { Pleistocene }\end{array}$ & lacunosa & $?$ & Gray & $\begin{array}{l}\text { Bioturbated } \\
\text { Blebs }\end{array}$ \\
\hline $\begin{array}{r}5-2, \infty 0 \\
a_{5-3,82}\end{array}$ & & & 2 & Grayish brown & Slightly smeared \\
\hline $\begin{array}{r}7-2,27 \\
a_{8-2,33}\end{array}$ & & & $?$ & Dark gray & Bioturbated \\
\hline $\begin{array}{r}\text { a } 8-2,33 \\
8-7,43\end{array}$ & & H. selii & 2 & Black & \\
\hline $\begin{array}{l}8-7,43 \\
9-2,40-90\end{array}$ & & $\frac{\text { H. selii }}{\text { C. mac. }}$ & 1 & Gray & \\
\hline $9-2,40-90$ & & $\begin{array}{c}\text { C. mac- } \\
\text { intyrei } \\
- \text { Fault }\end{array}$ & $?$ & Black & Bioturbated \\
\hline $10-1,96$ & & H. selii & 1 & Black & \\
\hline $11-3,20-30$ & & & $?$ & Gray & Crystal ash \\
\hline$a_{12-7,30}$ & & $\mathrm{CN} 12 \mathrm{~d}$ & 3 & Gray & \\
\hline $\mathrm{a}_{12, \mathrm{CC}}$ & & & 5 & Black & \\
\hline $13-3,100$ & & $\mathrm{CN} 12 \mathrm{~b}$ & $<1$ & Gray & 3 laminae \\
\hline $13-7,40$ & & & $<1$ & Gray & \\
\hline $15-5,120$ & & & $1-2$ & Gray & Bioturbated \\
\hline $16-1,50$ & & & $<1$ & Gray & Bioturbated \\
\hline$a_{16-2,50}$ & & $\mathrm{CN} 12 \mathrm{a}$ & 2 & Black & Bioturbated \\
\hline $16-3,28$ & late & & $<0.5$ & Gray & \\
\hline $\begin{array}{l}16-3,130 \\
16-4,90\end{array}$ & Pliocene & & $<0.5$ & Gray & \\
\hline $\begin{array}{l}16-4,90 \\
16-5,25\end{array}$ & & & $<0.5$ & Gray & Bioturbated \\
\hline $\begin{array}{l}16-5,25 \\
16-5,55\end{array}$ & & & $\begin{array}{l}<0.5 \\
<0.5\end{array}$ & Gray & Bioturbated \\
\hline $\begin{array}{c}16-5,55 \\
a_{16-5}, 142\end{array}$ & & & $<0.5$ & Gray & Bioturbated \\
\hline $\begin{array}{l}a_{16-5,142} \\
a_{16-6,3}\end{array}$ & & & $\begin{array}{l}7 \\
4\end{array}$ & Black & \\
\hline $\begin{array}{c}a_{16-6,3} \\
16-6,32\end{array}$ & & $\mathrm{CN} 11 \mathrm{~b}$ & $<1$ & Black & \\
\hline $\begin{array}{l}16-6,32 \\
16-6,80\end{array}$ & & & $<\begin{array}{r}1 \\
1\end{array}$ & Gray & Bioturbated \\
\hline $\begin{array}{l}16-6,80 \\
17-1,60\end{array}$ & & & $\begin{array}{l}1 \\
2\end{array}$ & Black & $\begin{array}{l}\text { Bioturbated } \\
\text { Tilted }\end{array}$ \\
\hline $\begin{array}{l}17-1,60 \\
17-2,100\end{array}$ & - & - & $?$ & Diack & Bioturbated \\
\hline $17-4,140$ & & & $?$ & & Bioturbated \\
\hline $17-5,60$ & & & $?$ & & Bioturbated \\
\hline $\mathrm{a}_{17-6,50}$ & & & ${ }_{1-2}^{2}$ & Gray & \\
\hline $17-6,118$ & & & $\begin{array}{l}1-2 \\
0-5\end{array}$ & Black & Bioturbated \\
\hline $\begin{array}{c}18-1,60 \\
a_{18-1,140}\end{array}$ & & CN11a & $\begin{array}{c}0-5 \\
7\end{array}$ & & Bioturbated \\
\hline $\begin{array}{l}a_{18-1}, 140 \\
18-2,90\end{array}$ & & & 7 & Black & Tilted \\
\hline $\begin{array}{c}18-2,90 \\
\mathrm{a}_{18-2,135}\end{array}$ & & & $\begin{array}{l}? \\
3\end{array}$ & & Bioturbated \\
\hline $\begin{array}{c}\mathrm{a}_{18-2}, 135 \\
18-4,35\end{array}$ & & & $?$ & Black & \\
\hline $\begin{array}{l}18-4,35 \\
18-4,65\end{array}$ & & & $?$ & & Bioturbated \\
\hline $\begin{array}{l}18-4,65 \\
18-5,55\end{array}$ & & & $?$ & & $\begin{array}{l}\text { Bioturbated } \\
\text { Bioturbated }\end{array}$ \\
\hline $\begin{array}{l}18-5,55 \\
19-1,10\end{array}$ & & $\mathrm{CN} 10 \mathrm{c}$ & $?$ & Black & $\begin{array}{l}\text { Bioturbated } \\
\text { Bleb }\end{array}$ \\
\hline $\begin{array}{l}19-1,10 \\
19-1,40\end{array}$ & Pliocene & & 1 & Black & Bleb \\
\hline $19-6,115$ & & $\begin{array}{l}\text { CN10b } \\
\text { - Fault }\end{array}$ & $1-2$ & Gray & $\begin{array}{l}\text { Dispersed by } \\
\text { burrowing }\end{array}$ \\
\hline$a_{20-1,119}$ & & & 7 & Black & \\
\hline${ }^{a} 20-3,15$ & & & 4 & Black & \\
\hline $20-3,77$ & & CNIIa & 1 & & Burrowed \\
\hline $20-5,15$ & & & $1-2$ & Gray & Bioturbated \\
\hline $20-6,135$ & & & $?$ & & Bioturbated \\
\hline $\begin{array}{l}20-7,3 \\
22-1,63\end{array}$ & & & $?$ & Gray & $\begin{array}{l}\text { Bioturbated } \\
\text { Bioturbated }\end{array}$ \\
\hline $\begin{array}{r}22-1,63 \\
22-3,70\end{array}$ & & CN1OC & $1-2$ & Gray & $\begin{array}{l}\text { Bioturbated } \\
\text { Bioturbated }\end{array}$ \\
\hline$a_{22-4,} 18$ & & —Fault & 2 & Gray & Bioturbated \\
\hline $23-2,80$ & & $\mathrm{CN} 9 \mathrm{~b}$ & $?$ & Gray & Bioturbated \\
\hline$a_{23-5,40}$ & & & 2 & Gray & Bioturbated \\
\hline $25-4,12$ & & & $?$ & Gray & Swirled and mixed \\
\hline $27-1,90$ & & & $?$ & & Bioturbated \\
\hline $27-2,80$ & & Barren & $?$ & & Bioturbated \\
\hline $27-4,40$ & & & $?$ & & Bioturbated \\
\hline $27-5,28$ & & & $?$ & & Bioturbated \\
\hline $27-6,140$ & & & $?$ & & Bioturbated \\
\hline $28-2,130$ & & & $?$ & & Bioturbated \\
\hline$a_{28-3}, 70$ & & $\mathrm{CN} 9 \mathrm{a}$ & 10 & Gray & Partially bioturbated \\
\hline $29-3,145$ & late & & $?$ & & Disturbed by rotation \\
\hline $29-5,75$ & Pliocene & & $?$ & & Burrowed \\
\hline $\begin{array}{l}30-1,2 \\
30-3,45\end{array}$ & & $\mathrm{CN} 9 \mathrm{~b}$ & $?$ & Black & Burrowed \\
\hline $\begin{array}{l}30-3,45 \\
31-5,30\end{array}$ & & & $?$ & & Bioturbated \\
\hline $31, \mathrm{CC}$ & & & $?$ & Black & Disturbed \\
\hline $32-1,150$ & & $\mathrm{CN} 12 \mathrm{a}$ & $?$ & & Bioturbated \\
\hline $32-4,20-50$ & & Small & ? & & Bioturbated zone \\
\hline $32-5,110$ & & $\leftarrow$ fault & $?$ & & Bioturbated \\
\hline$a_{32-6}, 143$ & & & 2.5 & Gray & Bioturbated top \\
\hline $33-3,80$ & & & $?$ & & Bioturbated \\
\hline$a_{33-3}, 135$ & & & 4 & Black & \\
\hline$a_{33-4,7}$ & & CNIIb & 7 & Black & \\
\hline $33-4,35$ & & & $?$ & & Bioturbated \\
\hline $33-4,65$ & & & $?$ & Black & $1-\mathrm{cm}$ bleb \\
\hline$a_{33-5,20}$ & & & Several & Gray & \\
\hline $34-1,35-50$ & & & ? & Gray & Mixed zone \\
\hline $34-1,90$ & & & $?$ & & Bioturbated \\
\hline $34-2,25$ & & & $?$ & & Bioturbated \\
\hline $34-2,56$ & & & $?$ & & Bioturbated \\
\hline $34-3,25$ & & & $?$ & & Bioturbated \\
\hline $34-3,40$ & early & & $?$ & & Bioturbated \\
\hline $34-3,65$ & Pliocene & & ? & & Bioturbated \\
\hline $34-4,25$ & Puocene & & ? & & Bioturbated \\
\hline $34-4,134$ & & CN11a & ? & & Bioturbated \\
\hline $34-5,15$ & & CNira & $?$ & & Bioturbated \\
\hline$a_{34-5,75}$ & & & 6 & Black & Tilted \\
\hline
\end{tabular}


Table 1. (Continued).

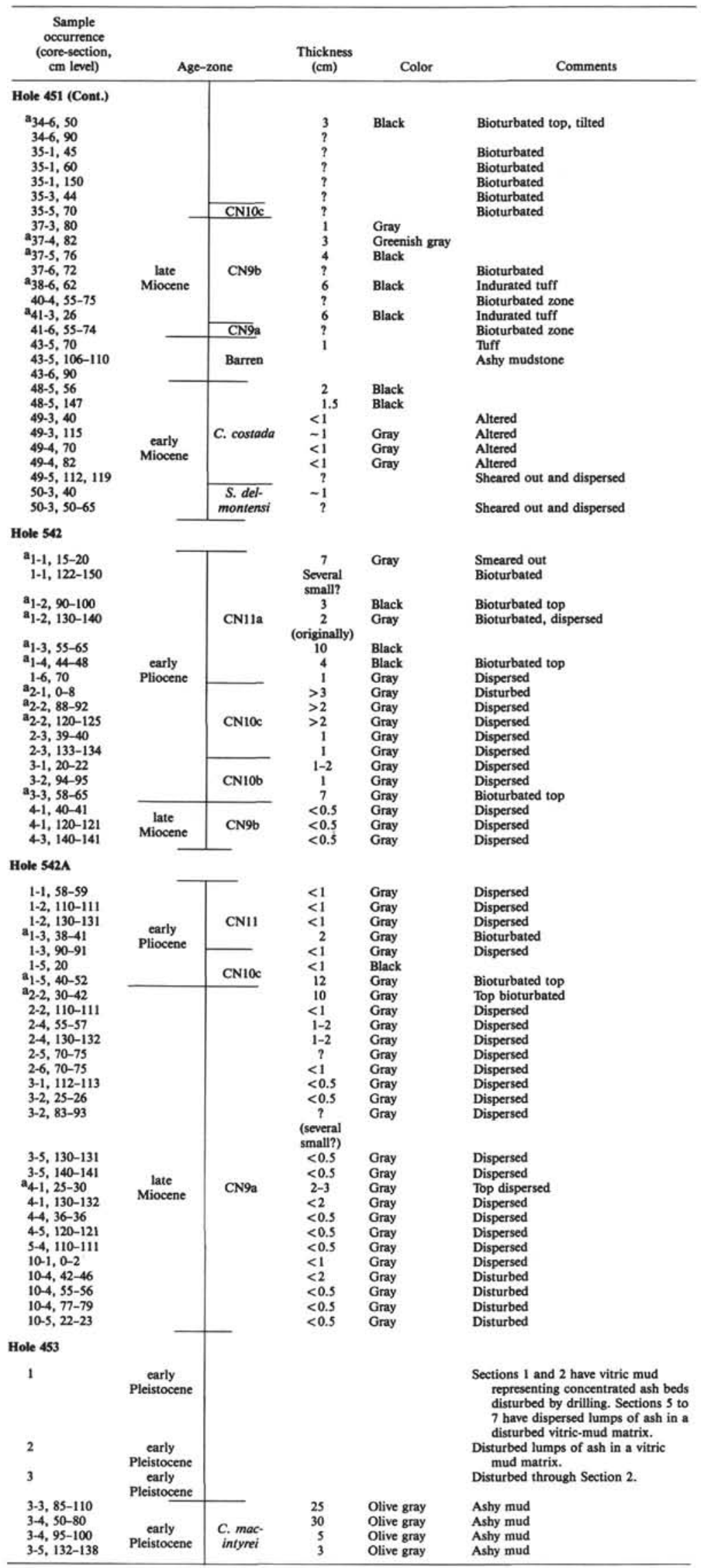


Table 1. (Continued).

\begin{tabular}{|c|c|c|c|c|c|}
\hline $\begin{array}{c}\text { Sample } \\
\text { occurrence } \\
\text { (core-section, } \\
\mathrm{cm} \text { level) }\end{array}$ & \multicolumn{2}{|c|}{ Age-zone } & \multirow[t]{2}{*}{$\begin{array}{l}\text { Thickness } \\
(\mathrm{cm})\end{array}$} & \multirow[t]{2}{*}{ Color } & \multirow[t]{2}{*}{ Comments } \\
\hline Hole 453 (Cont.) & & & & & \\
\hline $3-6,15$ & \multirow{17}{*}{$\begin{array}{c}\text { late } \\
\text { Pliocene }\end{array}$} & \multirow{14}{*}{$\mathrm{CN} 12 \mathrm{~d}$} & $<2$ & Olive gray & Ashy mud, disturbed \\
\hline $4-1,40-45$ & & & $3-4$ & Olive gray & Disturbed lump \\
\hline $4-1,55-61$ & & & $3 ?$ & Olive gray & Disturbed lump \\
\hline $4-1,73-9$ & & & $5-7$ & Olive gray & Disturbed lump \\
\hline $4-2,15-25$ & & & s? & Olive gray & Disturbed lump \\
\hline $4-3,35-60$ & & & $10 ?$ & Olive gray & Ashy mud; disturbed bed \\
\hline $\begin{array}{r}4-3,140 \text { to } \\
4-4,20\end{array}$ & & & $5 ?$ & Olive gray & Very disturbed \\
\hline $4-4,120$ & & & 2 & Olive gray & Disturbed \\
\hline $4-5,20-40$ & & & $?$ & Olive gray & Disturbed ashy mud \\
\hline$a_{4-5}, 62-75$ & & & 5 & Dark gray & Bioturbated top \\
\hline $4-5,95-100$ & & & $?$ & Olive gray & Ashy mud \\
\hline $4-6,20-30$ & & & ? & Olive gray & Disturbed lump \\
\hline $4-6,139-142$ & & & & Olive gray & Ashy mud \\
\hline $4-6,147-150$ & & & $?$ & Olive gray & $\begin{array}{l}\text { Ashy mud } \\
\text { Has dispersed lumps of ash in highly }\end{array}$ \\
\hline 5 & & $\mathrm{CN} 12 \mathrm{~b}$ & & & $\begin{array}{l}\text { Has dispersed lumps of ash in highly } \\
\text { deformed Sections } 1 \text { and } 2 \text {. }\end{array}$ \\
\hline $6-6,125$ & & \multirow{2}{*}{$\mathrm{CN} 12 \mathrm{a}$} & $<1$ & $\begin{array}{l}\text { Dusky yellow } \\
\text { green }\end{array}$ & Disturbed \\
\hline 7 & & & & & $\begin{array}{l}\text { Has dispersed lumps of ash in highly } \\
\text { deformed mud in Section } 1 \text {. }\end{array}$ \\
\hline$a_{7-3,113-116}$ & \multirow{10}{*}{$\begin{array}{c}\text { late } \\
\text { Miocene } \\
\text { to early } \\
\text { Pliocene }\end{array}$} & \multirow{10}{*}{$\begin{array}{c}\text { Forams } \\
\mathrm{N} 17-\mathrm{N} 20\end{array}$} & $2-3$ & $\begin{array}{l}\text { Very dark } \\
\text { gray }\end{array}$ & Bed \\
\hline $8-1,40-50$ & & & $?$ & Olive gray & Ashy mud \\
\hline $8-1,90-100$ & & & $?$ & Olive gray & Disturbed ashy mud \\
\hline$a_{8-1,148-150}$ & & & 2? & $\begin{array}{l}\text { Brownish } \\
\text { black }\end{array}$ & Lump \\
\hline$a_{8-2,8-10}$ & & & $1-2$ & $\begin{array}{l}\text { Brownish } \\
\text { black }\end{array}$ & Curved bed \\
\hline${ }^{a} 8-2,90-95$ & & & 4-5 & $\begin{array}{l}\text { Brownish } \\
\text { black }\end{array}$ & Bed \\
\hline $8-3,5-10$ & & & $?$ & $\begin{array}{l}\text { Olive gray } \\
\text { Olive gray }\end{array}$ & Ashy mud \\
\hline $\begin{array}{l}8-3,20-90 \\
8-5,82-85\end{array}$ & & & $\begin{array}{c}\text { Several } \\
?\end{array}$ & $\begin{array}{l}\text { Olive gray } \\
\text { Olive gray }\end{array}$ & $\begin{array}{l}\text { Lumps of ash this interval } \\
\text { Ashy mud }\end{array}$ \\
\hline $\begin{array}{l}8-5,82-85 \\
9-3,15-20\end{array}$ & & & 5 & Olive gray & Ashy turbidite \\
\hline 10 & & & & & $\begin{array}{l}\text { Has numerous ashy patches up to } \\
0.5 \mathrm{~cm} \text { scattered in Sections } 1\end{array}$ \\
\hline 11 & & \multirow{10}{*}{ Barren } & & & $\begin{array}{l}\text { to } 4 \text {. } \\
\text { Has dispersed ashy spots throughout } \\
\text { Sections } 1 \text { and } 2 \text {. }\end{array}$ \\
\hline$a_{11-3,127-131}$ & & & 3 & $\begin{array}{c}\text { Brownish } \\
\text { black }\end{array}$ & Undisturbed \\
\hline 12 & & & & & Has only dispersed ash, no beds. \\
\hline $13-3,100-110$ & & & ? & Greenish gray & Disturbed ashy mud \\
\hline $13-3,130-140$ & & & $?$ & Olive & Disturbed ashy mud \\
\hline $13-4,80-95$ & & & i & Greenish gray & Altered disturbed ashy mud \\
\hline $13-5,80-117$ & & & ? & Greenish gray & Altered disturbed ashy mud \\
\hline$a_{14-1,55-57}$ & & & $1-2$ & Black & Bed \\
\hline $14-1,74-75$ & & & 1 & Gray & Thin bed \\
\hline $\begin{array}{l}a_{14-2,50-55} \\
15 \text { and } 16\end{array}$ & & & 5 & Dark gray & $\begin{array}{l}\text { Bioturbated top } \\
\text { No distinct ash beds in relatively } \\
\text { undisturbed Cores } 15 \text { and } 16 .\end{array}$ \\
\hline$a_{17-1,130-135}$ & \multirow{4}{*}{$\begin{array}{l}\text { middle } \\
\text { Miocene }\end{array}$} & & 5 & Black & Bioturbated top \\
\hline$a_{17-1}, 142-144$ & & & 2 & Very dark gray & \\
\hline$a_{17-5}, 32-37$ & & D. alata & $2-5$ & Dark gray & Partly dispersed by bioturbation \\
\hline $\begin{array}{l}a_{18-1}, 57-58 \\
a_{18-1}, 106-108\end{array}$ & & & $<1$ & Black & \\
\hline $\begin{array}{l}a_{18-1}, 106-108 \\
a_{18-2,113-119}\end{array}$ & & & $<2$ & $\begin{array}{l}\text { Black } \\
\text { Black }\end{array}$ & \\
\hline $\begin{array}{l}\mathrm{a}_{18-2}, 113-119 \\
18-3,99-100\end{array}$ & & & $\begin{array}{l}2-3 \\
<1\end{array}$ & $\begin{array}{l}\text { Black } \\
\text { Gray }\end{array}$ & Partly dispersed by bioturbation \\
\hline$a_{18-3,125-128}$ & & & $1-2$ & Black & Dispersed by burrows \\
\hline$a_{18-5,50-56}$ & & & $2-3$ & Black & Partly dispersed by burrows \\
\hline $\mathrm{a}_{18-5,85-105}$ & early & & $?$ & Black & Abundant ash in drilling breccia \\
\hline$a_{18-6,80-82}$ & Miocene & C. costata & $1-2$ & Black & Dispersed by burrows \\
\hline$a_{19-1,81-83}$ & & & $1-2$ & $\begin{array}{c}\text { Brownish } \\
\text { black }\end{array}$ & Dispersed by burrows \\
\hline$a_{19-3,23-27}$ & & & $1-2$ & Black & Dispersed by burrows \\
\hline$a_{19-5,120-123}$ & & & $1-2$ & Olive black & Dispersed by burrows \\
\hline a $19-7,5-6$ & & & 1 & Dark gray & Dispersed by burrows \\
\hline $20-4,10-18$ & & & $2-3$ & Very dark gray & May be 2 redistributed \\
\hline 21 and 22 & Oligocene & & & & $\begin{array}{l}\text { No recovery. Below this, ash is virtu- } \\
\text { ally nonexistent, isolated occur- } \\
\text { rences as noted. }\end{array}$ \\
\hline $27-1,65-66$ & & & $<1$ & & Altered \\
\hline $32-4,66-68(?)$ & middle & & $<2$ & $\begin{array}{l}\text { Light greenish } \\
\text { gray }\end{array}$ & Feldspathic mudstone \\
\hline $34-2,42-43$ & Eocene & & 1 & Greenish gray & Altered \\
\hline Hole 543A & & & & & \\
\hline 1 & Quaternary & & & & Core 1 is the mud-line core-very \\
\hline $1-2,85-95$ & & & & Olive gray & $\begin{array}{l}\text { disturbed, generally vitric mud. } \\
\text { Ashy mud }\end{array}$ \\
\hline $1-2,131-137$ & & & 3-4 & $\begin{array}{l}\text { Light brownish } \\
\text { gray }\end{array}$ & May be Roseau ash \\
\hline $1-4,20-35$ & & & ? & Dark gray & Ashy mud \\
\hline $\begin{array}{l}2-1,86 \\
2-1,91\end{array}$ & & & & & Cores 2-10 are Eocene to Campanian, \\
\hline $2-2,50$ & middle & & & & $\begin{array}{l}\text { continuous in cored interval with } \\
\text { the cores of Hole } 543 \text {. Cores }\end{array}$ \\
\hline $2-2,60$ & Eocene & & & & $543 \mathrm{~A}-2,3$, and 5 contain tiny, \\
\hline $\begin{array}{l}2-2,61 \\
2 C C, 15\end{array}$ & & & & & horizontal, light greenish gray \\
\hline $3-1,21$ & & & & & $\begin{array}{l}\text { smectite-rich beds that are proba- } \\
\text { bly altered volcanic ash. All but }\end{array}$ \\
\hline $3-1,29$ & early & & & & one are less than $1 \mathrm{~cm}$ in thick- \\
\hline $\begin{array}{l}3-1,64 \\
5-2,31-33\end{array}$ & Eocene & & 2 & & ness. \\
\hline
\end{tabular}

Note: Color is indicated for unbioturbated and undisturbed beds.

a These ash beds $\geq 2 \mathrm{~cm}$ in thickness. They are the major ash beds of Figure 4 . 


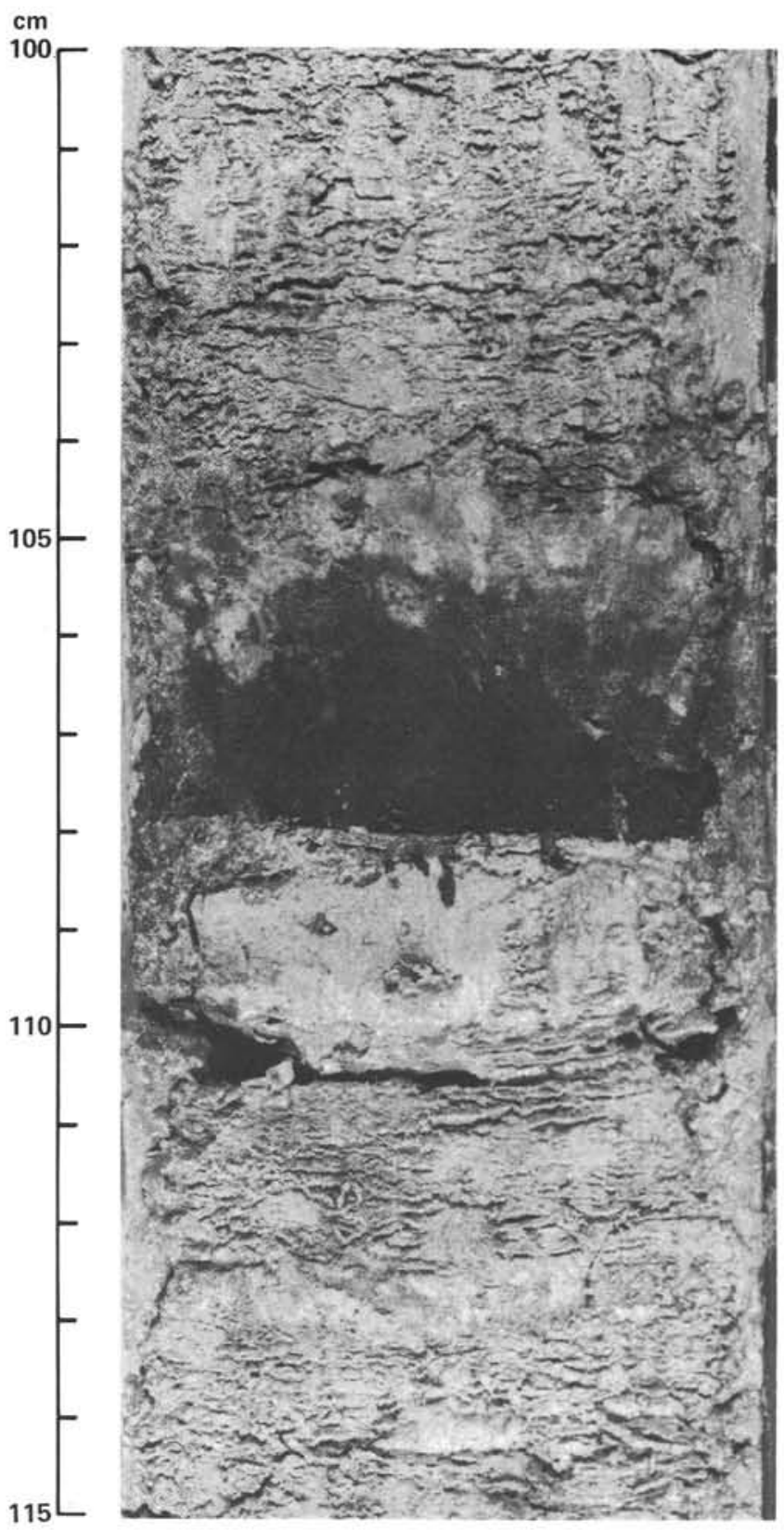

Figure 2. Photograph of a typical thick ash bed, with a flat bottom and bioturbated top (Sample 543-18-1, 100-115 cm).

Thunnell, 1977; Cadet and Fujioka, 1980; Cadet, Thisse, et al., 1982; Cadet, Pouclet, et al., 1982). This synchroneity supports a global, plate-tectonic control rather than a local control on arc magmatism (Kennett, 1981). The long hiatus in the late Miocene corresponds with a shift in Lesser Antilles volcanism in the northern part of the arc from sources in the Limestone Caribbees (Fig. 1) to sources in the more westerly arc of presently active volcanoes (Martin-Kaye, 1969; Tomblin, 1975; Westercamp, 1979).

Geochemical and mineralogical studies are currently in progress to evaluate the significance of the ash stratigraphy to the petrological evolution of the Lesser Antilles arc.

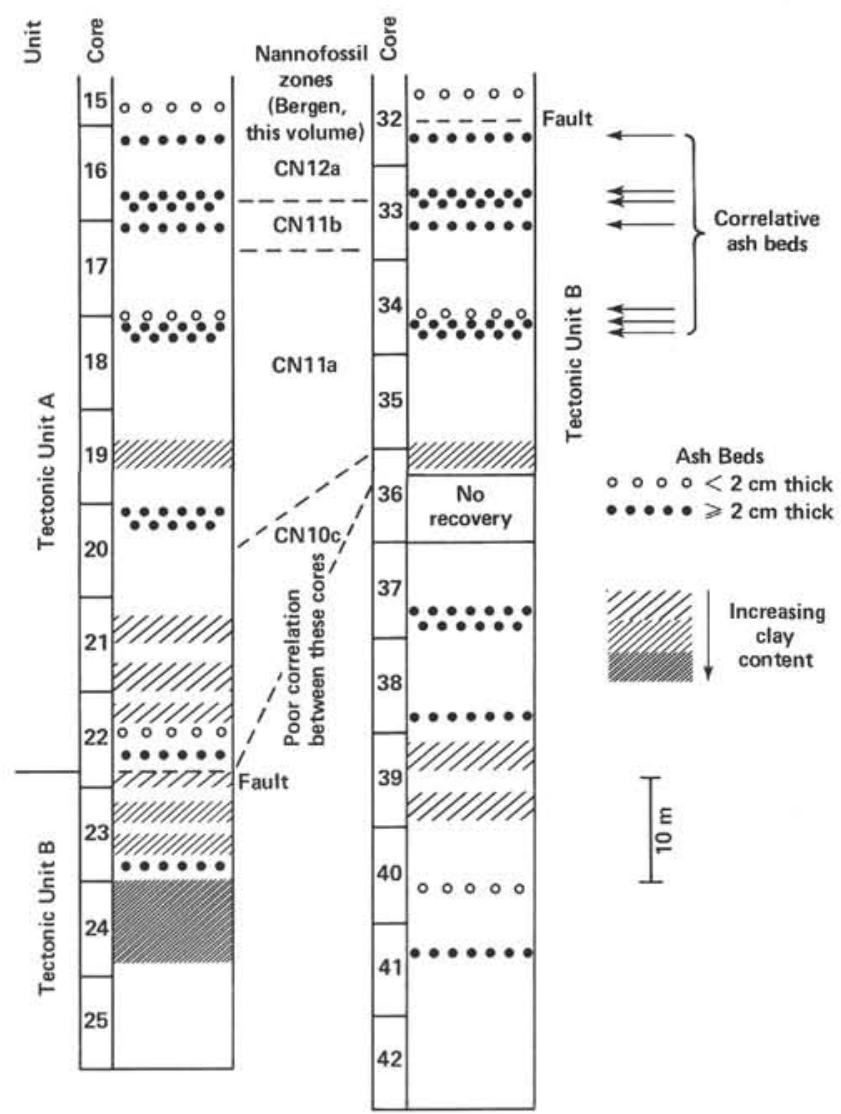

Figure 3. Correlation of Site 541 ash beds and nannofossil zones between portions of Tectonic Units A and B, which have been repeated by faulting. The correspondence was first suggested by the similarity in thickness and spacing of pairs of closely spaced ash beds near the base of Core 16 and the top of Core 33. Comparison of the sections above and below demonstrated that seven ash beds, altogether, could be matched in the two intervals. Nannofossil zonal boundaries are from Bergen (this volume).

\section{ACKNOWLEDGMENT}

I thank Bill Coulbourn for reviewing the manuscript and for suggesting that a bit more emphasis be placed on using ash beds to estimate offsets along faults.

\section{REFERENCES}

Bukry, D., 1975. Coccolith and silicoflagellate stratigraphy, northwestern Pacific Ocean, Deep Sea Drilling Project Leg 32. In Larson, R. L. Moberly, R., et al., Init. Repts. DSDP, 32: Washington (U.S. Govt. Printing Office), 677-692.

Cadet, J. P., and Fujioka, K., 1980. Neogene volcanic ashes and explosive volcanism: Japan Trench transect, Leg 57, Deep Sea Drilling Project. In Scientific Party, Init. Repts. DSDP, 56, 57, Pt. 2: Washington (U.S. Govt. Printing Office), 1027-1041.

Cadet, J. P., Pouclet, A., Thisse, Y., Bardintzeff, J. M., and Azéma, J., 1982. Middle America Neogene explosive volcanism and ash layers: evidence from the Middle America Trench transect, Deep Sea Drilling Project Leg 67. In Aubouin, J., von Huene, R., et al., Init. Repts. DSDP, 67: Washington (U.S. Govt. Printing Office), 475-489.

Cadet, J. P., Thisse, Y., Pouclet, A., Bardintzeff, J. M., and Stephan, J. F., 1982. Tephra from Deep Sea Drilling Project Leg 66: Middle America Trench Transect (southern Mexico). In Watkins, J., Moore, J. C., et al., Init. Repts. DSDP, 66: Washington (U.S. Govt. Printing Office), 687-695. 
Carey, S., and Sigurdsson, H., 1980. The Roseau ash: deep-sea tephra deposits from a major eruption on Dominica, Lesser Antilles arc. J. Volcanol. Geotherm. Res., 7:67-86.

Kennett, J. P., 1981. Marine tephrochronology. In Emiliani, C. (Ed.), The Sea (Vol. 7): New York (John Wiley \& Sons), 1373-1436.

Kennett, J. P., and Thunnell, R., 1977. On explosive Cenozoic volcanism and climatic implications. Science, 196:1231-1234.

Kling, S. A., 1982. Radiolarians from the Mariana Trough and Trench region: Deep Sea Drilling Project Leg 60. In Hussong, D. M., Uyeda, S., et al., Init. Repts. DSDP, 60: Washington (U.S. Govt. Printing Office), $537-555$.

Martin-Kaye, P. H. A., 1969. A summary of the geology of the Lesser Antilles. Overseas Geol. Miner. Resour., 10:172-206.

Sigurdsson, H., Sparks, R. S. J., Carey, S., and Huang, T. C., 1980. Volcanogenic sedimentation in the Lesser Antilles arc. J. Geol., $88: 523-540$.
Tomblin, J. F., 1975. The Lesser Antilles and Aves Ridge. In Nairn, A. E. M., and Stehli, F. V. (Eds.), The Ocean Basins and Margins (Vol. 3): New York (Plenum), 467-500.

Westbrook, G. K., 1982. The Barbados Ridge Complex: tectonics of a mature forearc system. In Leggett, J. K. (Ed.), Trench-Forearc Geology: Sedimentation and Tectonics on Modern and Ancient Active Plate Margins. Spec. Publ. Geol. Soc. London: Oxford (Blackwell Scientific Publications), pp. 275-290.

Westercamp, D., 1979. Diversité, contrôle structural et origines du volcanism récent dans l'arc insulair des Petites Antilles. Bull. Bur. Rech. Geol. Min. Sect. 4, pp. 211-226.

Date of Initial Receipt: October 28, 1983

Date of Acceptance: October 28, 1983

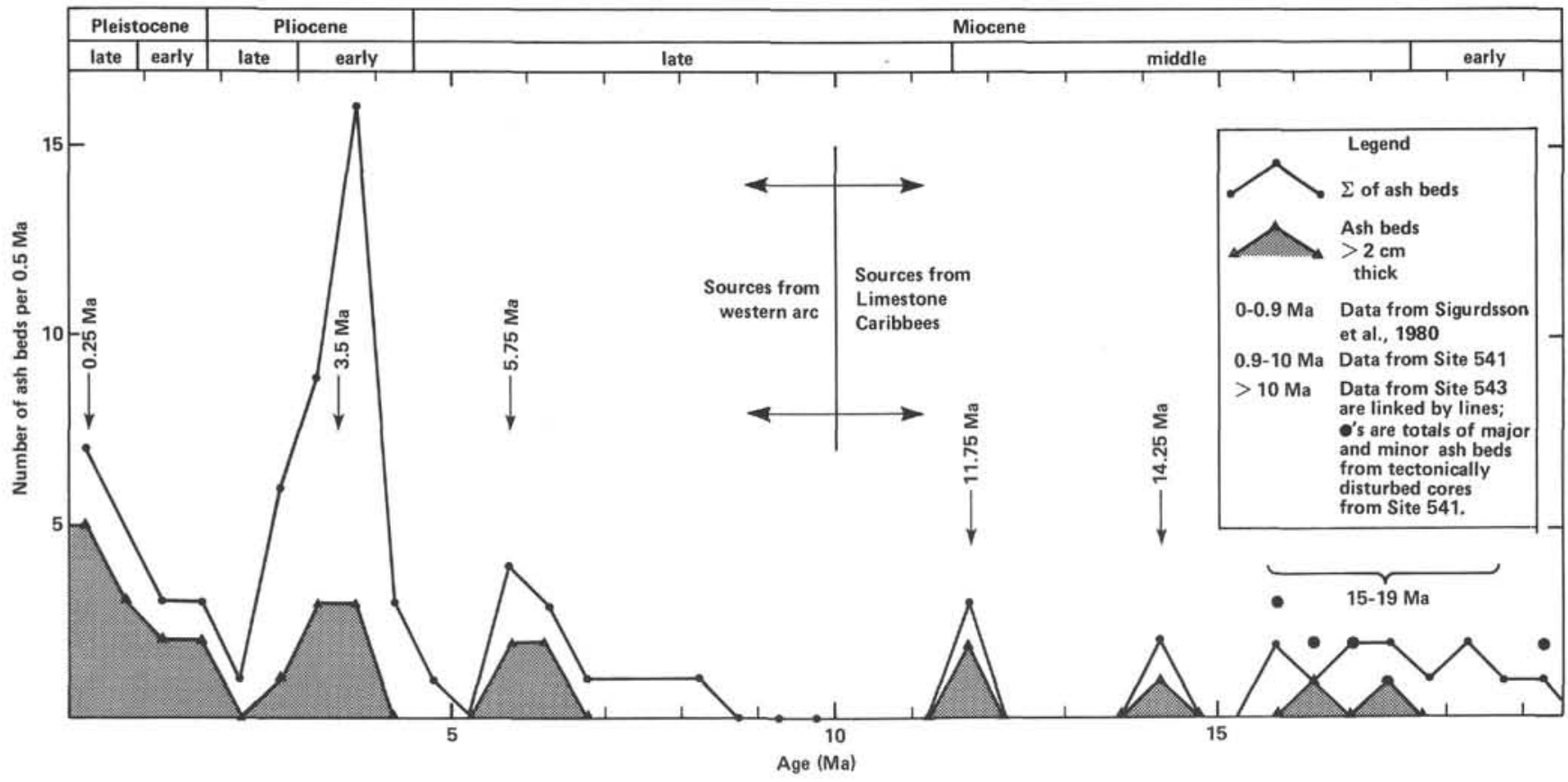

Figure 4. Abundances of ash beds (number per $0.5 \mathrm{Ma}$ ) plotted versus absolute age. Stippled pattern represents number of major ash beds ( $\geq 2 \mathrm{~cm}$ thick). 